\title{
Global Consensus Position Statement on the Use of Testosterone Therapy for Women
}

\author{
Susan R. Davis, ${ }^{1, A}$ Rodney Baber, ${ }^{2, A, B}$ Nicholas Panay, ${ }^{3, A}$ Johannes Bitzer, ${ }^{4, C}$ \\ Sonia Cerdas Perez, ${ }^{5, D}$ Rakibul M. Islam, ${ }^{1, A}$ Andrew M. Kaunitz, ${ }^{6, E}$
} Sheryl A. Kingsberg, ${ }^{7, F}$ Irene Lambrinoudaki, ${ }^{8, G}$ James Liu, ${ }^{9, E}$ Sharon J. Parish, ${ }^{10, H}$ JoAnn Pinkerton, ${ }^{11,{ }^{\prime}}$ Janice Rymer, ${ }^{12,1}$ James A. Simon, ${ }^{13,14, \mathrm{H}}$ Linda Vignozzi, ${ }^{15,16, \mathrm{C}}$ and Margaret E. Wierman ${ }^{17,5}$

'Women's Health Research Program, School of Public Health and Preventive Medicine, Monash University,
Melbourne, Victoria 3004, Australia; ${ }^{2}$ University of Sydney, Sydney 2006, Australia; ${ }^{3}$ Queen Charlotte's \&
Chelsea and Westminster Hospitals and Imperial College, London SW7 2AZ, United Kingdom; ${ }^{4}$ University
Hospital Basel, 4031 Basel, Switzerland; ${ }^{5}$ Endocrinology Department, Hospital Cima and University of Costa
Rica, San Jose 10201, Costa Rica; ${ }^{6}$ Department of Obstetrics and Gynecology, University of Florida College
of Medicine, Jacksonville, Florida $32209 ;{ }^{7}$ Behavioral Medicine, University Hospitals Cleveland Medical
Center and Case Western Reserve University School of Medicine, Cleveland, Ohio $44106 ;{ }^{8}$ Medical School,
National and Kapodistrian University of Athens, 11528 Athens, Greece; ${ }^{9}$ Department of Obstetrics and
Gynecology, University Hospitals Cleveland and Case Western Reserve University School of Medicine,
Cleveland, Ohio $44106 ;{ }^{10}$ Weill Cornell Medical College, New York, New York $10065 ;{ }^{11}$ Department of
Obstetrics and Gynecology, the University of Virginia Health System, Charlottesville, Virginia $22903 ;{ }^{12}$ King's
College London, Guy's and St. Thomas' Foundation Hospital Trust, London SE1 9RT, United Kingdom;
${ }^{13}$ George Washington University, Washington, DC $20052 ;{ }^{14}$ IntimMedicine Specialists, Washington, DC
20036; ${ }^{15}$ Department of Biomedical, Experimental and Clinical Sciences "Mario Serio," University of
Florence, 50121 Florence, Italy; ${ }^{16}$ Careggi Hospital, 50134 Florence, Italy; ${ }^{17}$ Department of Medicine,
Integrative Physiology and Obstetrics and Gynecology, University of Colorado, Anschutz Medical Campus,
Colorado 80045

Author society affiliations: ${ }^{A}$ The International Menopause Society; ${ }^{B}$ Royal Australian and New Zealand College of Obstetricians and Gynaecologists; ${ }^{C}$ The International Society for Sexual Medicine; ${ }^{D}$ The Federacion Latinoamericana de Sociedades de Climaterio y Menopausia; ${ }^{E}$ The American College of Obstetricians and Gynecologists; ${ }^{F}$ The North American Menopause Society; ${ }^{G}$ The European Menopause and Andropause Society; ${ }^{H}$ The International Society for the Study of Women's Sexual Health; 'The Royal College of Obstetricians and Gynaecologists; and 'The Endocrine Society

ORCiD numbers: 0000-0002-2955-0415 (S. R. Davis).

This Position Statement has been endorsed by the International Menopause Society, The Endocrine Society, The European Menopause and Andropause Society, The International Society for Sexual Medicine, The International Society for the Study of Women's Sexual Health, The North American Menopause Society, The Federacion Latinoamericana de Sociedades de Climaterio y Menopausia, The Royal College of Obstetricians and Gynecologists, The International Society of Endocrinology, The Endocrine Society of Australia, and The Royal Australian and New Zealand College of Obstetricians and Gynecologists. * (J Clin Endocrinol Metab 104: 4660-4666, 2019)

ISSN Print 0021-972X ISSN Online 1945-7197 Printed in USA

Copyright @ 2019 The Author(s), Published by the Endocrine Society

This article has been published under the terms of the Creative Commons Attribution Non-Commercial, No-Derivatives License (CC BY-NC-ND; https://creativecommons.org/ licenses/by-nc-nd/4.0/)

Received 15 July 2019. Accepted 16 July 2019.

First Published Online 2 September 2019 *This Statement is being simultaneously published in the journals Climacteric, Maturitas,
Journal of Sexual Medicine, and Journal of Clinical Endocrinology and Metabolism on
behalf of the International Menopause Society, The European Menopause and Andro-
pause Society, The International Society for Sexual Medicine, and The Endocrine Society,
respectively.
Abbreviations: FSAD, female sexual arousal disorder; FSD, female sexual dysfunction;
HSDD, hypoactive sexual desire disorder/dysfunction; RCT, randomized controlled trial. 
$\mathbf{T}$ here are no clearly established indications for testosterone therapy for women. Nonetheless, clinicians have treated women with testosterone for decades, with the intention of alleviating a variety of symptoms, with uncertain benefits and risks. In most countries, testosterone therapy is prescribed off-label such that women are using either testosterone formulations approved for men with dose modification, or compounded therapies. Because of these issues, there is a compelling case for a global consensus Position Statement on testosterone therapy for women based on the available evidence from placebo/comparator randomized controlled trials (RCTs).

This Position Statement was developed, by consensus between the participating organizations, to inform health care professionals of the known benefits and potential risks of testosterone therapy for women. The aims were to provide clear guidance as to which women might benefit from testosterone therapy, to identify symptoms, signs, and conditions for which evidence does not support the prescribing of testosterone, to explore areas of uncertainty, and to identify any prescribing practices that have the potential to cause harm.

\section{Methods}

A Task Force of representatives of leading societies, whose international memberships include clinicians assessing and managing sex steroid therapy for women, was established. The Task Force agreed on the issues that needed to be addressed, after which a systematic review and meta-analysis of the benefits and risks of testosterone therapy for women were conducted (1). The Task Force then met on 17 May 2019 in Berlin, Germany, and drafted this consensus position statement.

Recommendations regarding the benefits and risks of testosterone therapy are based on findings from blinded placebo/ comparator RCTs, of at least 12 weeks duration for which data were available for inclusion in meta-analyses (1). The findings are reported with Levels of evidence and Grades of Recommendations (2). Clinical practice recommendations are agreed expert opinions of the panel. Through constructive discussion, unanimous consensus agreement was reached on all the Expert Opinion recommendations included here.

\section{Recommendations}

\section{Measurement of testosterone, female sexual dysfunction, and endogenous androgen levels}

(1) Recommendations pertaining to the measurement of circulating testosterone in women

(a) Testosterone may act directly via the androgen receptor/nongenomic androgenic action, or by reduction to the more potent androgen dihydrotestosterone and/or aromatization to estradiol and their metabolites. (b) Testosterone concentrations decline during the reproductive years $(3,4)$ (Level IIB).

(c) Testosterone concentrations appear to be maintained in women beyond the age of 65 years, but whether this confers a benefit is yet to be understood $(3,5)$ (Level IIB).

(d) Total testosterone can be measured with high accuracy and reproducibility using liquid/gas chromatography and tandem mass spectrometry assays (6) (Grade B).

(e) Direct assays for the measurement of total and free testosterone are highly unreliable in the female range $(6,7)$ (Grade A).

(f) Reference laboratories should be "harmonized" with biological standards in coordination with the US Centers for Disease Control and Prevention (8) (Expert Opinion).

(g) Measurement of testosterone using direct assays in clinical practice is appropriate, if liquid/gas chromatography and tandem mass spectrometry assay is not available, to exclude high baseline concentrations and also to exclude supraphysiological concentrations during treatment (Expert Opinion).

(h) Current research into testosterone physiology and clinical effects should mainly focus on measuring total testosterone as the main biomarker rather than "free" testosterone because evidence that "free" testosterone is the biologically active testosterone fraction is lacking (9) (Expert Opinion).

(2) Recommendations for the terminology for female sexual dysfunction (FSD)

(a) Hypoactive sexual desire disorder/dysfunction (HSDD) and female sexual arousal disorder (FSAD) are distinct conditions that should be categorized separately when considering the impact of androgens on their clinical presentation and response to treatment (Grade B).

(b) Although HSDD and FSAD overlap, they have distinct etiologies, risk factors, clinical features, and responses to psychological and biological interventions (10) (Grade B).

(c) Traditional specifiers (i.e., lifelong vs acquired; generalized vs situational) should be retained and used to further categorize and stratify treatments for HSDD and other female sexual disorders/dysfunctions (10).

(d) The diagnosis of HSDD in clinical practice should be based on thorough clinical assessment (11) guided by available diagnostic criteria such as the International Society for the 
Study of Women's Sexual Health $(12,13)$ or the International Classification of Diseases, 11th edition (14) (Expert Opinion).

(3) Recommendations pertaining to the associations between endogenous androgen concentrations and female sexual function

(a) The associations between endogenous androgen concentrations and sexual function in women remain uncertain because of issues relating to the sensitivity and specificity of androgen assays in some studies and insufficient data (Insufficient).

(b) The physiology of androgens is complex because of their conversion in tissues and possible intracrine metabolism in multiple tissues (Insufficient).

(c) No cutoff blood level can be used for any measured circulating androgen to differentiate women with and without sexual dysfunction (15) (Grade C).

\section{Recommendations regarding systemic testosterone therapy for postmenopausal women, in doses that approximate physiological testosterone concentrations for premenopausal women, based on findings from meta-analyses of placebo/comparator-controlled RCTs $(1,16)$}

There are insufficient data to make any recommendations regarding the use of testosterone in premenopausal women for treatment of sexual function or any other outcome (Insufficient).

(4) Recommendations regarding testosterone treatment of naturally or surgically postmenopausal women with HSDD, with/or without concurrent estrogen therapy

(a) Testosterone therapy, in doses that approximate physiological testosterone concentrations for premenopausal women, exerts a beneficial effect on sexual function including increases, above the effects of placebo/comparator therapy, of an average of one satisfying sexual event per month, and increases in the subdomains of sexual desire, arousal, orgasmic function, pleasure, and sexual responsiveness, together with a reduction in sexual concerns including sexual distress (Level I, Grade A).

(b) Because the majority of studies reporting on sexual function recruited women assessed as having HSDD or generalized FSD, these recommendations cannot be generalized to other subtypes of FSD or women without sexual dysfunction (Expert Opinion). (c) The recommendations of 4(a) do not apply to injectables, pellets, or formulations that result in supraphysiological blood concentrations of testosterone, or compounded preparations (Expert Opinion).

(5) Recommendations regarding the effects of testosterone on wellbeing, mood, and cognition in postmenopausal women

(a) There is insufficient evidence to support the use of testosterone to enhance cognitive performance, or to delay cognitive decline, in postmenopausal women (Insufficient).

(b) Available data show no effect of testosterone therapy on general wellbeing (Level I, Grade A).

(c) Testosterone may improve wellbeing in premenopausal women but data are inconclusive (Level 1, Grade B).

(d) Available data do not show an effect of testosterone on depressed mood (Level I, Grade B).

(6) Recommendations regarding the musculoskeletal effects of testosterone

(a) Few studies have evaluated the musculoskeletal effects of testosterone.

(b) Of the studies that have reported musculoskeletal outcomes, the number of included participants has been small, all participants were taking concurrent estrogen therapy, and no studies have been in women with osteoporosis.

(c) The available data do not support an effect of testosterone treatment on bone mineral density at the spine, total hip, or femoral neck at 12 months (Level I, Grade A).

(d) No statistically significant effect of testosterone administered in physiologic doses has been demonstrated on lean body mass, total body fat, or muscle strength (Level I, Grade A).

(e) There is a need for clinical trials to evaluate the impact of testosterone treatment on musculoskeletal tissues (Expert Opinion).

(7) Recommendations regarding possible androgenic side effects of testosterone therapy

(a) Systemic testosterone therapy for postmenopausal women, in doses that approximate physiological testosterone concentrations for premenopausal women, is associated with mild increases in acne and body/facial hair growth in some women, but not with alopecia, clitoromegaly, or voice change (Level I, Grade A). 
(8) Recommendations regarding testosterone therapy and cardiovascular health

(a) Oral testosterone therapy is associated with adverse lipid profiles with negative effects on high-density lipoprotein-cholesterol and lowdensity lipoprotein-cholesterol levels, and is not recommended (Level I, Grade A).

(b) Studies of nonoral testosterone therapies (percutaneous and injectable), in doses that approximate physiological testosterone concentrations for premenopausal women, have shown no statistically significant adverse effects on lipid profiles over the short term (Level I, Grade A).

(c) Testosterone therapy has not been associated with increases in blood pressure, blood glucose, or HbA1c levels (Level I, Grade A).

(d) A nonsignificant trend for an increased risk of deep venous thrombosis has been seen with testosterone therapy; however, the role of concurrent estrogen therapy in possible venous thrombosis risk cannot be excluded (Level I, Grade A).

(e) Limited data preclude assessment of the effects of testosterone therapy on myocardial infarction or death (Insufficient data).

(f) RCTs of testosterone therapy have excluded women at high cardiometabolic disease risk; most have included women taking concurrent estrogen therapy, and all have been of relatively short duration. Therefore, recommendations regarding the effect of physiologic doses of testosterone in postmenopausal women on cardiovascular health are not generalizable to a more "at-risk" population or to long-term therapy.

(9) Recommendations regarding testosterone therapy and breast health

(a) Testosterone therapy does not increase mammographic breast density (Level I, Grade A).

(b) Available data suggest that short-term transdermal testosterone therapy does not impact breast cancer risk (Level I, Grade A).

(c) Data from RCTs are insufficient to assess longterm breast cancer risk (Insufficient data).

(d) There are no data to support the use of testosterone therapy to prevent breast cancer (Insufficient data).

(e) Women with a prior diagnosis of breast cancer were excluded from the randomized trials for HSDD. Caution is recommended for testosterone use in women with hormonesensitive breast cancer (Expert Opinion).

(10) Recommendations regarding testosterone therapy and serious adverse events

(a) Testosterone therapy for postmenopausal women, in doses that approximate physiological testosterone concentrations for premenopausal women, is not associated with serious adverse events (Level I, Grade A).

(b) Because RCTs of testosterone therapy have excluded women at high cardiometabolic disease risk, and most have included women taking concurrent estrogen therapy, recommendation $10(a)$ is not generalizable to a more "at-risk" population (Expert Opinion).

(c) Safety data for testosterone in physiologic doses are not available beyond 24 months of treatment (Level I, Grade A).order

\section{Clinical care of postmenopausal women}

(11) Recommendations regarding full assessment of FSD before commencing testosterone therapy

(a) FSD including HSDD, FSAD, and orgasmic disorder/dysfunction have multiple etiologies including biopsychosocial factors such as neuroendocrine imbalance, physical ill health or disease, interpersonal difficulties, psychological distress, and sexually repressive cultural or religious values (Grade C).

(b) Treatments should follow this biopsychosocial model and include pharmacologic options (hormone therapies and other pharmacologic agents), psychotherapy, or multimodal treatments that combine both (17) (Grade B).

(12) Recommendations regarding current testosterone therapy and postmenopausal women

(a) The only evidence-based indication for the use of testosterone in women is for the treatment of postmenopausal women who have been diagnosed as having HSDD after formal biopsychosocial assessment (Level I, Grade A).

(b) There is an unmet need for the provision and approval of testosterone treatments specific to women, formulated with the aim of approximating physiological testosterone concentrations for premenopausal women (Expert Opinion). 
(c) Where an appropriate approved female testosterone preparation is not available, off-label, prescribing of an approved male formulation is reasonable, provided hormone concentrations are maintained in the physiologic female range (Expert Opinion).

(d) Compounded "bioidentical" testosterone therapy cannot be recommended for the treatment of HSDD because of the lack of evidence for efficacy and safety, unless an authorized equivalent preparation is not available (Expert Opinion). In the absence of an available approved product, if a compounded product is needed, the compounding pharmacy should be compliant with purity of Active Pharmaceutical Ingredients and Good Manufacturing Practice to meet industry standards for quality and safety. Dosing should be limited to achieving testosterone concentrations in the physiologic premenopausal range.

(e) Use of any testosterone preparation that results in supraphysiologic concentrations of testosterone, including pellets and injections, is not recommended (Expert Opinion).

(f) Should a trial of testosterone therapy be given for HSDD, a baseline total testosterone concentration should be measured before commencement, with a repeat level 36 weeks after treatment initiation (Level IIA, Grade C).

(g) Patients should be monitored for their clinical response to treatment and assessed for signs of androgen excess with a serum total testosterone level every 6 months, to screen for overuse (Expert Opinion).

(h) If no benefit is experienced by 6 months, treatment should be ceased (Level IB, Grade C).

(13) Recommendations regarding other androgenic preparations

(a) Systemic dehydroepiandrosterone is not associated with significant improvement in libido or sexual function in postmenopausal women with normal adrenal function and cannot be recommended for women with HSDD (18) (Level IA, Grade A).

(b) In the absence of vulvovaginal atrophy, vaginal dehydroepiandrosterone has not been tested and thus cannot be recommended for treatment of HSDD (Expert Opinion).
(14) Recommendations regarding the design of future trials of physiologically dosed testosterone (Expert Opinion for all)

(a) More adequately powered, double-blind RCTs, without selection bias and with consistent reporting of standardized outcomes, are needed to comprehensively establish the benefits and risks of testosterone therapy for women.

(b) For studies of testosterone and FSD:order

(i) Relief of the distress associated with sexual dysfunction is a primary aim of FSD treatment.

(ii) Presently, no questionnaire covers all domains of female sexual function such that a combination of domains from different questionnaires should be used.

(iii) Satisfying sexual events should no longer be used as a primary efficacy measurement in clinical trials of women with FSD.

(iv) A set of clearly defined core outcomes needs to be established.

(v) There is a need for an instrument to assess sexual function with the following characteristics: general applicability; not disease-specific; high discriminant validity between women diagnosed with FSD and sexually functional women; validated, to measure FSD per se and as an instrument to screen for and diagnose FSD and demonstrating clinically meaningful response to intervention; cover different domains, with each domain comprising several items; translated and backtranslated in a variety of languages, satisfies the most stringent assessment to gain approval by regulatory agencies.

(c) There is a need for adequately powered RCTs of the effects of testosterone on the musculoskeletal health of women with normal bone mass, low bone mass, osteopenia/osteoporosis, and sarcopenia, with outcomes including vertebral and total hip and femoral neck bone mineral density, trabecular bone score, serum biomarkers, fracture risk, body composition, and muscle strength.

(d) There is a need for adequately powered RCTs of the effects of testosterone on cognitive performance. 
(e) Studies must be undertaken to establish the longer term cardiometabolic and breast safety of testosterone therapy for women.

\section{Summary and Key Messages}

The international panel concluded the only evidencebased indication for testosterone therapy for women is for the treatment of HSDD, with available data supporting a moderate therapeutic effect. There are insufficient data to support the use of testosterone for the treatment of any other symptom or clinical condition, or for disease prevention.

Meta-analyses of the available data show no severe adverse events during physiological testosterone use, with the caveat that women at high cardiometabolic risk were excluded from study populations. The safety of long-term testosterone therapy has not been established.

It was considered of utmost importance that the diagnosis of HSDD involves a full clinical assessment and that other factors contributing to FSD must be identified and addressed before testosterone therapy is initiated $(10,11)$. A blood total testosterone level should not be used to diagnose HSDD. Treatment should only be with formulations that achieve blood concentrations of testosterone that approximate premenopausal physiological concentrations. Because no approved female product is presently approved by a national regulatory body, male formulations can be judiciously used in female doses and blood testosterone concentrations must be monitored regularly. The panel recommended against the use of compounded testosterone.

The panel highlighted the pressing need for more research into testosterone therapy for women and the development and licensing of products indicated specifically for women.

\section{Acknowledgments}

The authors thank Lee Tomkins, Executive Director, International Menopause Society, for her assistance in the planning and coordination of the consensus meeting.

Financial Support: The development of the Position Statement was supported by the Societies listed on the first page of this article. There is no other external funding to report. S.R.D. is a National Health and Medical Research Council Senior Principal Research Fellow (grant no. 1135843).

Author Contributions: Systematic review and metaanalysis of the literature: R.M.I. and S.R.D.; additional literature search: all coauthors; synthesis of information and draft statements: all coauthors; first manuscript draft: S.R.D., R.B., N.P.; manuscript review: all coauthors.
Correspondence and Reprint Requests: Susan R. Davis, MBBS, PhD, Women's Health Research Program, School of Public Health and Preventive Medicine, Monash University, 553 St. Kilda Road, Melbourne, Victoria 3004, Australia. E-mail:susan.davis@monash.edu.

Disclosure Summary: S.R.D. receives funding from a National Health and Medical Research Council Project Grant (no. 1105305), a National Breast Foundation accelerator grant, and the Grollo-Ruzzene Foundation; reports honoraria from Besins and Pfizer Australia; has been a consultant to Besins Healthcare, Mayne Pharmaceuticals, Lawley Pharmaceuticals and Que Oncology; and is an investigator for Que Oncology (money paid to her institution). R.B. has received funding from Que Oncology for a clinical research trial and has received honoraria or consultation fees from Besins Pharmacology and Pfizer Australia for educational lectures and participated in speaker's bureaus for Besins Pharma and Pfizer. J.B. reports having received honoraria or consultation fees from Bayer AG, Libbs, Gedeon Richter, Jenapharm, Ava, Natural Cycles, Exeltis, Theramex, Mithra, Effik, Merck, and Mitsubishi, and has participated in company-sponsored speaker's bureaus for Bayer AG, Libbs, Gedeon Richter, Jenapharm, Ava, Exeltis, Theramex, and Effik. S.A.K. is a consultant who has participated in investigator or on scientific advisory boards for AMAG, Daré, Duchesney, Emotional Brain, Valeant, Endoceutics, IVIX, Palatin Technologies, and Mitsubishi; has stock options with Viveve; is in receipt of grants/research support from Endoceutics and Palatin; receives honoraria from the above listed and has participated in a company-sponsored speaker's bureau for TherapeuticsMD. J.L. has received funding from AbbVie, AMAG, and Femsys for clinical trials and reports having received honoraria or consultation fees as a consultant to Allergan, TherapeuticsMD, Ferring, Daré, and Mitsubishi-Tanabe. N.P. has received funding from Abbot/ Mylan (OPTIMISE study), Asarina (SEPRANOLONE study), Lawley Pharmaceuticals (T-BONE study), Pharm Olam/NeRRe (SWITCH 1 study), PregLem (ESMYA study), and Yes Company (REVIVE Me study); received honoraria or consultation fees from Abbott, Bayer, Besins, Glenmark, Kora, Meda, Mithra, MSD, Mylan, Novo Nordisk, Pfizer, SeCur, and Shionogi; and has participated in company-sponsored speaker's bureaus for Abbott, Bayer, Besins, Glenmark, Meda, MSD, Mylan, Novo Nordisk, Pfizer, Shionogi, and Theramex. S.J.P. reports having received honoraria or consultation fees for participating in Scientific Advisory Boards for Allergen, AMAG, Valeant, and Duchesnay Pharmaceuticals and scientific consultancy for AMAG, Daré Bioscience, JDS Therapeutics, Strategic Science Technologies, Proctor and Gamble, and TherapeuticsMD; has participated in sponsored speaker's bureaus for AMAG Pharmaceuticals-two lectures, and Valeant Pharmaceuticals-two lectures; and her partner is the recipient of a Valeant Pharmaceuticals unrestricted grant to develop HSDD educational materials. J.R. is a recipient of a KHP/GSTT Grant, Rosetree's Foundation Grant, and a 100,000 Genomes Project Grant and reports having received honoraria from Gilead to be on an advisory board. J.A.S. has received funding from AbbVie, Inc., Allergan, Plc, Agile Therapeutics, Bayer 
Healthcare LLC, Endoceutics, Inc., GTx, Inc., Ipsen, Myovant Sciences, New England Research Institute, Inc., ObsEva SA, Palatin Technologies, Symbio Research, Inc., TherapeuticsMD, and Viveve Medical; reports having received honoraria or consultation fees from AbbVie, Inc., Allergan, Plc, AMAG Pharmaceuticals, Inc., Amgen, Ascend Therapeutics, Bayer HealthCare Pharmaceuticals Inc., CEEK Enterprises, LLC, Covance Inc., Daré Bioscience, Duchesnay USA, Hologic Inc., KaNDy/NeRRe Therapeutics Ltd., Mitsubishi Tanabe Pharma Development America, Inc., ObsEva SA, Sanofi S.A., Shionogi Inc., Sprout2 Inc., and TherapeuticsMD; participation in speaker's bureaus for AbbVie, Inc., AMAG Pharmaceuticals, Inc., Duchesnay USA, Novo Nordisk, and Shionogi Inc.; and is a stock shareholder of Sermonix Pharmaceuticals (Columbus, $\mathrm{OH})$ (direct purchase). L.V. reports an affiliation or financial interest with TEVA-Theramex for scientific support, Bayer for scientific support and consultancy activity, and IBSA for scientific support. M.E.W. has received funding from: VA Merit Review 001 9U54AG062319-06 CO-SCORE, Corcept Therapeutics, Inc., Novartis LC1699C2301, and Cancer League of Colorado; has received honoraria or consultation fees from Pfizer to review ASPIRE grant applications for studies of acromegaly and an Endocrine Society honorarium for teaching in the Endocrine board review and Clinical Endocrine Update; and is a consultant to the National Hockey League and a consultant to the UDADA US Anti-doping Association. There are no other potential conflicts to declare.

Data Availability: Data sharing is not applicable to this article as no datasets were generated or analyzed during the current study.

\section{References and Notes}

1. Islam MR, Bell RJ, Green S, Page M, Davis SR. Efficacy and safety of testosterone therapy for women: a systematic review and metaanalysis of randomized controlled trails. Lancet Diabetes Endocrinol. In press.

2. Shekelle PG, Woolf SH, Eccles M, Grimshaw J. Developing clinical guidelines. West J Med. 1999;170(6):348-351.

3. Davison SL, Bell R, Donath S, Montalto JG, Davis SR. Androgen levels in adult females: changes with age, menopause, and oophorectomy. J Clin Endocrinol Metab. 2005;90(7):3847-3853.

4. Haring R, Hannemann A, John U, Radke D, Nauck M, Wallaschofski H, Owen L, Adaway J, Keevil BG, Brabant G. Agespecific reference ranges for serum testosterone and androstenedione concentrations in women measured by liquid chromatographytandem mass spectrometry. J Clin Endocrinol Metab. 2012; 97(2):408-415.

5. Cappola AR, Ratcliffe SJ, Bhasin S, Blackman MR, Cauley J, Robbins J, Zmuda JM, Harris T, Fried LP. Determinants of serum total and free testosterone levels in women over the age of 65 years. J Clin Endocrinol Metab. 2007;92(2):509-516.

6. Wang C, Catlin DH, Demers LM, Starcevic B, Swerdloff RS. Measurement of total serum testosterone in adult men: comparison of current laboratory methods versus liquid chromatographytandem mass spectrometry. J Clin Endocrinol Metab. 2004; 89(2):534-543.
7. Groenestege WM, Bui HN, ten Kate J, Menheere PP, Oosterhuis WP, Vader HL, Heijboer AC, Janssen MJ. Accuracy of first and second generation testosterone assays and improvement through sample extraction. Clin Chem. 2012;58(7):1154-1156.

8. Rosner W, Vesper H; Endocrine Society; American Association for Clinical Chemistry; American Association of Clinical Endocrinologists; Androgen Excess/PCOS Society; American Society for Bone and Mineral Research; American Society for Reproductive Medicine; American Urological Association; Association of Public Health Laboratories; Endocrine Society; Laboratory Corporation of America; North American Menopause Society; Pediatric Endocrine Society. Toward excellence in testosterone testing: a consensus statement. J Clin Endocrinol Metab. 2010;95(10): $4542-4548$.

9. Goldman AL, Bhasin S, Wu FCW, Krishna M, Matsumoto AM, Jasuja R. A reappraisal of testosterone's binding in circulation: physiological and clinical implications. Endocr Rev. 2017;38(4): 302-324.

10. Clayton AH, Goldstein I, Kim NN, Althof SE, Faubion SS, Faught BM, Parish SJ, Simon JA, Vignozzi L, Christiansen K, Davis SR, Freedman MA, Kingsberg SA, Kirana PS, Larkin L, McCabe M, Sadovsky R. The International Society for the Study of Women's Sexual Health Process of Care for Management of Hypoactive Sexual Desire Disorder in Women. Mayo Clin Proc. 2018;93(4): 467-487.

11. Simon JA, Davis SR, Althof SE, Chedraui P, Clayton AH, Kingsberg SA, Nappi RE, Parish SJ, Wolfman W. Sexual well-being after menopause: an International Menopause Society White Paper. Climacteric. 2018;21(5):415-427.

12. Parish SJ, Meston CM, Althof SE, Clayton AH, Goldstein I, Goldstein SW, Heiman JR, McCabe MP, Segraves RT, Simon JA. Toward a more evidence-based nosology and nomenclature for female sexual dysfunctions-part III. J Sex Med. 2019;16(3): 452-462.

13. Parish SJ, Goldstein AT, Goldstein SW, Goldstein I, Pfaus J, Clayton AH, Giraldi A, Simon JA, Althof SE, Bachmann G, Komisaruk B, Levin R, Spadt SK, Kingsberg SA, Perelman MA, Waldinger MD, Whipple B. Toward a more evidence-based nosology and nomenclature for female sexual dysfunctions-part II. J Sex Med. 2016;13(12):1888-1906.

14. Sexual dysfunctions, in 17 conditions related to sexual health. ICD11 for Mortality and Morbidity Statistics. Available at: https:// icd.who.int/browse11/l-m/en\#/http \%3a \% 2f\%2fid.who.int $\% 2$ ficd \%2fentity\%2f160690465. Accessed 22 July 2019.

15. Davis SR, Davison SL, Donath S, Bell RJ. Circulating androgen levels and self-reported sexual function in women. JAMA. 2005; 294(1):91-96.

16. Achilli C, Pundir J, Ramanathan P, Sabatini L, Hamoda H, Panay N. Efficacy and safety of transdermal testosterone in postmenopausal women with hypoactive sexual desire disorder: a systematic review and meta-analysis. Fertil Steril. 2017;107: 475-482.

17. Kingsberg SA, Althof S, Simon JA, Bradford A, Bitzer J, Carvalho J, Flynn KE, Nappi RE, Reese JB, Rezaee RL, Schover L, Shifrin JL. Female sexual dysfunction-medical and psychological treatments, committee 14 (published correction appears in J Sex Med. 2018; 15(2):270). J Sex Med. 2017;14(12):1463-1491.

18. Elraiyah T, Sonbol MB, Wang Z, Khairalseed T, Asi N, Undavalli C, Nabhan M, Altayar O, Prokop L, Montori VM, Murad MH. Clinical review: the benefits and harms of systemic dehydroepiandrosterone (DHEA) in postmenopausal women with normal adrenal function: a systematic review and meta-analysis. J Clin Endocrinol Metab. 2014;99(10):3536-3542. 